\title{
Development of License Plate Recognition on Complex Scene with Plate-Style Classification and Confidence Scoring Based on KNN
}

\author{
Vince Jebryl MONTERO $^{\dagger \text { a) }}$, Member and Yong-Jin JEONG ${ }^{\dagger}$, Nonmember
}

\begin{abstract}
SUMMARY This paper presents an approach for developing an algorithm for automatic license plate recognition system (ALPR) on complex scenes. A plate-style classification method is also proposed in this paper to address the inherent challenges for ALPR in a system that uses multiple plate-styles (e.g., different fonts, multiple plate lay-out, variations in character sequences) which is the case in the current Philippine license plate system. Methods are proposed for each ALPR module: plate detection, character segmentation, and character recognition. K-nearest neighbor $(\mathrm{KNN})$ is used as a classifier for character recognition together with a proposed confidence scoring to rate the decision made by the classifier. A small dataset of Philippine license plates but with relevant features of complex scenarios for ALPR is prepared. Using the proposed system on the prepared dataset, the performance of the system is evaluated on different categories of complex scenes. The proposed algorithm structure shows promising results and yielded an overall accuracy higher than the existing ALPR systems on the dataset consisting mostly of complex scenes.

key words: plate detection, segmentation, character recognition, $K$-nearest neighbors (KNN)
\end{abstract}

\section{Introduction}

Automatic license plate recognition (ALPR) is a major aspect in intelligent transportation system (ITS) and appears in a variety of applications, including traffic surveillance, traffic enforcement, automatic toll collection, security control and parking management systems [1], [2]. Such system heavily relies on image processing techniques and computer vision algorithms to identify and recognize license plates rather than manual human observation [2].

ALPR pipeline is typically divided into three main modules: Plate Localization, Plate Segmentation, and Character Recognition where each module plays an important role in the accuracy of the final output [3]. Though being a mature technology, there are several challenges with currently deployed ALPR systems such as scalability (deploying the same system to different sites) and jurisdictional plate-style variations (each country or state has different license plate styles and formats). Such challenges in ALPR are the motivation for the continued research and development.

In Philippine transport setting where multiple variation of license plate-style exists for different generation of license plates and some are very old and degraded license

Manuscript received February 10, 2018.

Manuscript revised July 17, 2018.

Manuscript publicized August 24, 2018.

${ }^{\dagger}$ The aurhors are with Dept. of Electronics and Communications Engineering, Kwangwoon University, Seoul, South Korea.

a) E-mail: vjm753@yahoo.com

DOI: 10.1587/transinf.2018EDP7060 plates especially to public utility vehicles are still widely used up to this day. These existing variants of Philippine license plate-styles poses a general difficulty in a number of commercial software that have been developed for ALPR without the prior knowledge of the currently used platestyles. Majority of these ALPR systems are particularly suited to their own country's license plate format and until now there is no recognized Philippine license plate recognition system from both international and national patent websites [2]. In this paper, an approach is presented for automatic license plate recognition system that is capable of handling the challenges posed by the current Philippine license plate system and as a reference contribution for the further development in the field of intelligent transport systems.

\section{License Plate Detection}

License plate detection is based on a multi-sensory structure where each sensor works on distinguishing a particular feature in the domain space (e.g., colors, edges, blobs, contours). The multi-sensory structure takes advantage of analyzing multiple domain spaces which increases the rate of detection. A total of three parallel sensors are utilized: plate character sensor for typical illumination, plate character sensory for dim and contrasting illumination, and a yellow plate sensor. Each sensor plays a significant role in detection of a candidate region and most importantly in the later stages of the pipeline where a candidate is given a proper pre-processing prior to character segmentation and character recognition and the binarization quality of a valid candidate region that will heavily influence the final output. Utilization of a multi-sensory license plate detection might seem heavy on the processor but as long as these sensors rely only on primitive features such as geometry, contrast, and color, it can be implemented efficiently by existing implementations of image processing techniques and machine vision algorithms. Implementation of this algorithm is done in $\mathrm{C}++$ using OpenCV 3.3.0 library with AMD Ryzen 7 1700 processor where a maximum CPU utilization below 20 percent is observed in testing an image with high definition resolution of $2048 \times 1080$.

\subsection{Sensor 1}

This sensor detects plate characters under typical illumination. Preprocessing is done to the colored image by convert- 


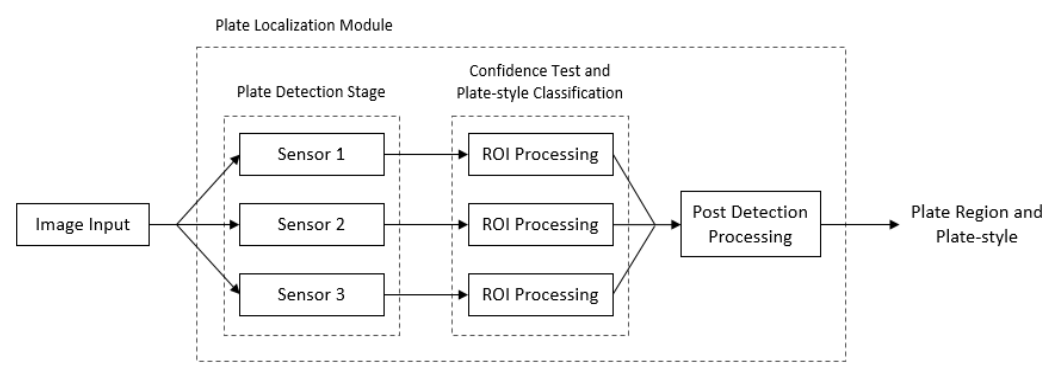

Fig. 1 Block diagram of multi-sensory plate localization module.

ing it to grayscale and applying Gaussian filter to smoothen the image. Then, binarization is done by applying a local adaptive threshold with a small window size. This is to minimize the effects of shadows and uneven illumination on the plate region which may cause loss of information for the proceeding stages.

After the preprocessing stage, a connected component analysis technique is applied to detect possible plate character contour filtered by contour point count, aspect ratio and by comparing the minimum rectangle area of fit with respect to the upright rectangle area of fit tangent to the contour point. License plate characters exhibits a minimal difference between the two rectangle areas.

Selective clustering algorithm is applied to the filtered contours. Contours that are similar in size, aspect ratio and relatively not very scattered from each other are clustered together. Then, clusters are filtered by cluster size. For complex and noisy background scene, it is common to have more than four clusters. Further filtering of clusters is necessary to reduce the processing time of the system. The proceeding filters are as follows:

1. Filter the cluster by the average aspect ratio of the elements. Above or below the threshold is discarded.

2. Compute the squared deviation with respect to the the vertical center $\left(y_{i}\right)$ of the rectangle boundary of each $N$ elements to the center line of the minimum area rectangle (MAR) of the cluster.

Let: $m=\tan (\theta)$ be the slop of MAR

$b=y_{c t r}-m x_{c t r} ;\left(x_{c t r}, y_{c t r}\right)$ is the center of MAR

$\left(x_{i}, y_{i}\right)$ be the center point of rectangular boundary

$S q D e v=\sqrt{\frac{1}{N} \sum_{1}^{N}\left(y_{i}-\left(m x_{i}+b\right)\right)^{2}}$

Then, set a maximum allowable threshold of $S q D e v$ and discard the cluster whose SqDev is above the threshold.

3. Filter the remaining candidates by setting a minimum and maximum aspect ratio of the cluster's MAR.

\subsection{Sensor 2}

This sensor detects plate characters under dim and high contrast illumination. The processing pipeline is identical to the Sensor 1 except that prior to the grayscale conversion,

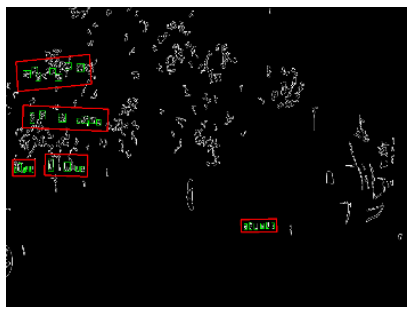

(a)

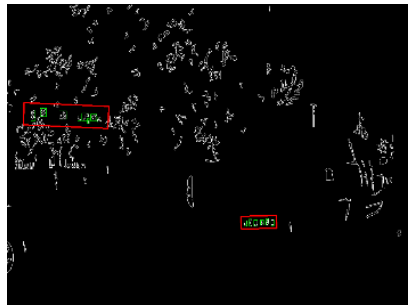

(b)
Fig. 2 (a) Potential clusters from a complex scene. (b) Filtered clusters.

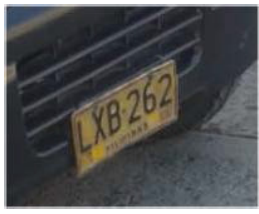

(a)

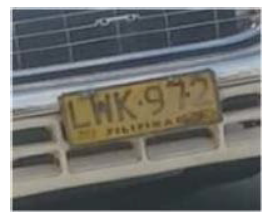

(b)

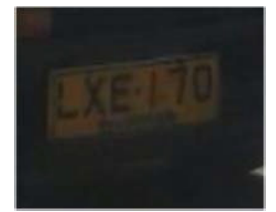

(c)
Fig. 3 (a) Dusty yellow plate. (b) Yellow plate that exhibits fading. (c) Heavily shadowed yellow plate that exhibits thinning of characters,

the contrast and brightness of the image is adjusted so that the image becomes brighter and contrast between darker and brighter parts of the image becomes more distinguishable than the original input image. This brightness and contrast adjustment will have a significant impact on the proceeding stages. Previously undetected character regions can now be detected and form a valid cluster to become a plate candidate.

\subsection{Sensor 3}

This sensor aims to detect yellow plates which is the color of public utility vehicles. Generally, public utility vehicle's license plates are assumed to be dirty or dusty because they run around all day in the busy urban streets with high exposure to smoke and dust. Most of these plates are very old and the quality of the characters in the plate are quite degraded and broken characters are fairly common. Dirt around the borders may cause these plate characters to be connected to the borders during binarization. For these reasons, Sensor 1 and Sensor 2 fails to detect most of these public utility vehicle plates.

Contrast and brightness of the input image is adjusted to minimize the effects of dust and make the plate characters 
more distinguishable. Then, the image is then filtered so that only those objects with a yellow color range are retained. An Otsu thresholding is applied after applying Box blur to the yellow filtered image to close the gaps between the character and border areas in the plate region [8]. This will make the license plate region more rectangular in shape. Then, a connected component analysis is applied and the following filters are used to locate plate candidate regions: minimum area threshold, aspect ratio, and area difference of the connected component with respect to its minimum rectangle of fit. An observed threshold of $30 \%$ area difference is set as a threshold to detect a highly probable candidate.

\section{Confidence Test and Plate-Style Classification}

The plate confidence test aims to determine the probability of a plate candidate region to match the horizontal projection of a license plate while identifying the plate-style will determine the structure of character sequence in a license plate during character recognition. Both methods of plate confidence testing and plate-style classification take advantage of the typographical structure of a license plate (e.g., spacing, gap width, character width, and positioning).

\subsection{Vertical Correction}

Vertical correction is the removal of top and bottom regions, e.g., screws, stickers and local region identification marks. Removal of these unwanted regions is a significant step for the proceeding stage. The first phase of the process is to create a vertical profile (Fig. 4) of the candidate region. This is done by counting the pixel changes from 0 to 1 or from 1 to 0 and is performed through all the rows of the candidate region. Then, a Gaussian profile (Fig. 4) consisting of values from a Gaussian distribution is used to mask the vertical profile. This will effectively heighten the values in middle region while lowering the values on the top and bottom regions. The masked vertical profile is then normalized to create a correction profile (Fig. 4). It is done by computing the average of the values obtained from masking then setting it as a threshold where values that are lower than the average is set to zero else to one. The correction profile is applied to the rows of the binarized plate image leaving behind only the region that contains the license plate characters.

\subsection{Horizontal Projection and Feature Extraction}

The vertically corrected image is resized to 125 x 50 pixels. A horizontal projection is obtained by accumulating the number of white pixel per column where the characters
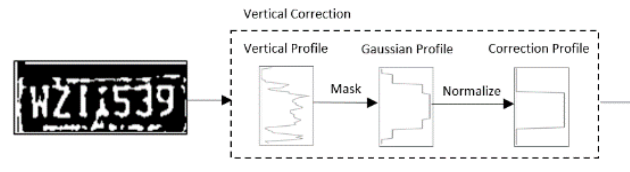

Fig. 4 are labeled as with white pixels while the background is labeled with black pixels. The integer sequence chain (ISC) is then generated from the horizontal projection and is composed of interchanging values of negative and positive integers. The positive integers represent the character projection while the negative integers represent the spacing projection where $125=\sum_{i=1}^{\mathrm{N}}\left|x_{i}\right| ; x_{i} \in$ ISC. Then, six features are extracted based from the ISC to be used as parameters in the confidence test described as follows:

a) Total Space per Octal (TSO): This feature is the sum of the absolute value of negative integers divided by eight.

$$
T S O=\frac{1}{8} \sum \text { |negtive integer } \mid
$$

b) Valid Plate Spacing (VPS): This feature is the accumulation of integers in between $(-1)$ to $(-18)$. The threshold of allowable valid spacing is observed among variety of samples.

c) Wide Element Count (WEC): This feature is the accumulation of positive integers between $(+9)$ to $(+22)$. The upper threshold is determined to consider possible connected characters. The lower threshold is the observed lower boundary of narrow element such as the presence of the character "I" or " 1 ".

d) Narrow Element Count (NEC): This feature is the accumulation of positive integers from $(+4)$ to $(+8)$ and indicate the presence of the characters " $I$ " and " 1 ".

e) Total Valid Elements (TVE): This feature is the sum of WEC and NEC.

f) Standard Deviation of Valid Elements (SDE): The standard deviation is the measure of how closely related are the valid elements.

$$
S D E=\sqrt{\frac{1}{N} \sum_{1}^{N}\left(x_{i}-\operatorname{Ave}(X)\right)}
$$

Where $x_{i}$ is an integer value belonging to set of integer values of valid elements and $\operatorname{Ave}(X)$ is the average of the integer values of valid elements.

\subsection{Plate-Style Classification}

The plate-style classification will determine the structure of character sequence in a license plate during the character recognition stage. Plate-styles are classified based on the typographical structure of the current Philippine license plates which exploits the length and positioning of the space separating the character groups (Fig. 6). The plate-style categories are as follows:

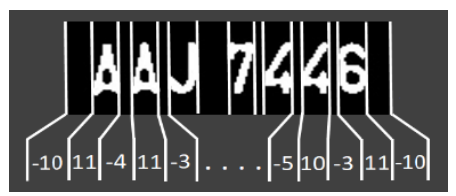

Fig. 5 Illustration of the integer sequence chain. 


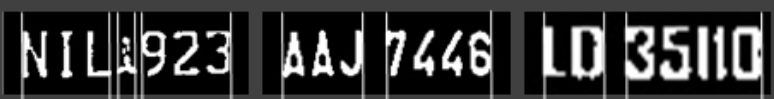

Fig. 6 Typographic differences of the length and positioning of the space separating the character groups of different plate-styles.

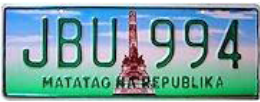

(a)

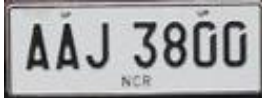

(d)

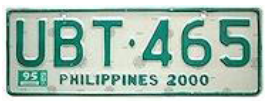

(b)

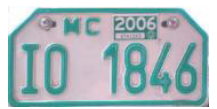

(e)

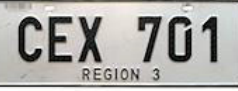

(c)

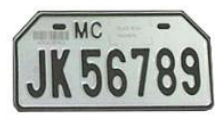

(f)
Fig. 7 License plate-styles. (a) Old plate-style on complex background - Plate-style 1. (b) Old plate-style on plain background - plate-style 1. (c) New generation old plate-style - Plate-style 1. (d) New plate-style plate-style 2 (e) Motorcycle old plate-style - plate-style 3. (f) Motorcycle new plate-style - plate-style 4 .

1. Not a recognizable plate - Plate-style 0: The candidate region will no longer proceed to the next phase.

2. Old plate-style for four-wheel vehicles - Plate-style 1: It is composed of a total of six characters, three letters and three numbers. (Fig. 7-a, b, c).

3. New plate-style for four-wheel vehicles - Plate-style 2: It is composed of a total of seven characters, three letters and four numbers. (Fig. 7-d).

4. Old plate-style for motorcycles - Plate-style 3: It is composed of a total of six characters, two letters and four numbers (Fig. 7-e).

5. New plate-style for motorcycles - Plate-style 4: It is composed of a total of seven characters, two letters and five numbers (Fig. 7-f).

\subsection{Confidence Test}

Confidence Test of a candidate region involves the features extracted from the ICS in which the six features are used to evaluate the confidence formula and the plate-style classification is used to determine whether or not to proceed with evaluation. The confidence formula and activation functions are as follows:

Let: $A=\mathrm{TSO}, B=\mathrm{VPS}, C=\mathrm{WEC}, D=\mathrm{NEC}, E=\mathrm{TVE}$, $F=\mathrm{SDE}$

$$
\begin{aligned}
Y_{A} & =\frac{1}{24}(4.9-|4.9-A|)^{2} ; A=\left\{\begin{array}{l}
A, \text { if } A<9.8 \\
9.8, \quad \text { else }
\end{array}\right. \\
Y_{B} & =e^{-(0.43|7.5-B|)^{8}} \\
Y_{C} & =e^{-(0.25|7.0-C|)^{8}} \\
Y_{D} & =\frac{1}{\left(1+e^{2 D-10}\right)}
\end{aligned}
$$

$$
\begin{aligned}
& Y_{E}=e^{-(0.50|7.3-E|)^{8}} \\
& Y_{F}=\frac{1}{\left(1+e^{1.8 F-10}\right)} \\
& \text { Confidence }=15 Y_{A}+20 Y_{B}+10 Y_{C}+10 Y_{D} \\
& \quad+25 Y_{E}+20 Y_{F}
\end{aligned}
$$

The activation functions $\left(Y_{A}, Y_{B}, \ldots, Y_{F}\right)$ are used to imitate neuron activation in an artificial neural network that instead of using a step function, it is more effective to use a continuous function to resolve partly overlapping features of classes. Constant values that are used in each activation function are derived from the observed typographical structure of the Philippine license plates. The weights of each activation function from the confidence formula are determined experimentally to come up with the most likelihood of optimal values.

\section{Post Detection Processing}

Post detection processing is a necessary stage to avoid overlapping of valid candidates from the multi-sensory plate detection structure. If an overlapping of candidate regions is detected from multiple sensors then a sensor priority based decision is made. The sensor priority is as follows: First priority - Sensor 3, Second priority - Sensor 1 and Third priority - Sensor 2. This is logically justified that if sensor 3 and sensor 1 or sensor 2 detected the same candidate region then the detected plate is a yellow plate and the necessary pre-segmentation processing for a yellow plate should be done. A logical justification also holds true that if sensor 1 and sensor 2 detected the same candidate region then the candidate region must be detectable under typical illumination. Brightness and contrast adjustment might affect its binarization quality.

\section{License Plate Character Segmentation}

License plate region is preprocessed before proper character segmentation to enhance the pixel density of the character regions. Image regions that are too thin might cause a problem for character recognition. Each plate region from different sensors are preprocessed differently. Then, a five phase character segmentation is applied to extract the character regions.

\subsection{Plate Region Preprocessing}

The candidate preprocessing from the confidence test removes most of the effects of shadow and high contrast regions but greatly erodes the character pixel region. This is done to accurately locate the character region for vertical correction, plate-style classification and confidence scoring. For sensor 1, the plate region is preprocessed by applying a Gaussian blur and local adaptive thresholding whose parameters differ from the previous stages. Plate region for sensor 2 is preprocessed by applying a brightness and contrast correction prior to applying the same preprocessing stages from 
sensor 1. Yellow plates from sensor 3 are preprocessed by applying a contrast limited adaptive histogram equalization (CLAHE) before subjected to the same preprocessing from sensor 1 to reduce the effects of blurring caused by dirt and enhances faded characters [8].

\subsection{Character Segmentation}

Character segmentation involves connected components analysis of the plate region. It is one of the most widely used approach for various segmentation methods [3], [6]. Clustering of the character regions involves a clustering method that groups together blobs with comparable geometric parameters (e.g., width, height, aspect ratio) and are adjacent to each other. Then, a threshold based from the widest and tallest regions is selected to detect probable broken characters. Plate characters tend to have uniform height and width with only a small deviation. Each broken character is clustered to form a single region

In mid-stage processing (Fig. 8), character regions are rearranged from left to right based on the $\mathrm{x}$-component of their rectangular bound. Then, small and thin individual regions that are under a certain threshold are removed. These regions might be dashes, background noises or border noises.

Segmentation overlapping is usually caused by dirt that are enhanced during binarization, and skewed portions, then is fixed by chopping off the overlapped region from the larger segment of the two neighboring regions. Connected characters are detected if the aspect ratio of the segment is less than 1.00 because Latin based characters typically have an aspect ratio greater than 1.40 . The region with connected characters is segmented by dividing the region into equal parts such that each new region will have an aspect ratio greater than 1.00 .

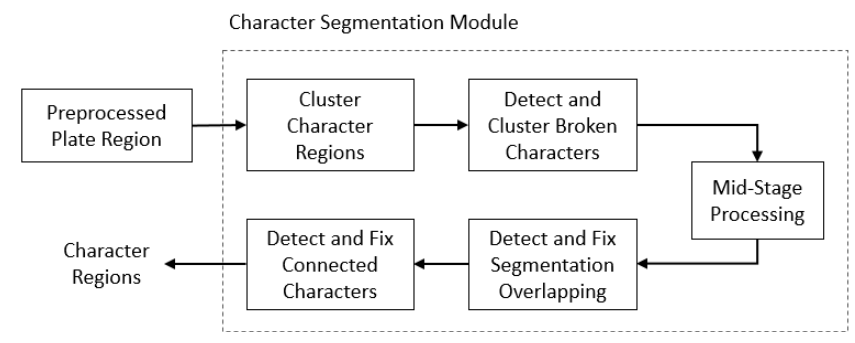

Fig. 8 Pipeline of character segmentation module

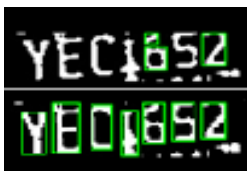

(a)

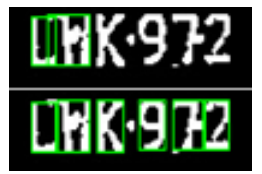

(b)

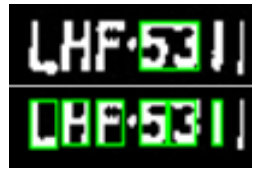

(c)
Fig. 9 (a) Segmentation with broken character. (b) Segmentation with overlapped regions. (c) Segmentation with connected characters.

\section{License Plate Character Recognition}

\subsection{K-Nearest Neighbor (KNN) as Character Classifier}

$\mathrm{KNN}$ is an effective and widely used classifier in the industry despite is simplicity [4], [5]. It also has high fault tolerance over non-linear multiclass problems because it does not assume any models for the distribution of feature vectors in space [5], [8], [14]. KNN computes the distance from an unknown to all samples in the template space and remembers the minimum distance [5], [8], [14], [17]. The class that has the minimum distance from the unknown is the nearest neighbor $k=1$ where the value $k$ refers to the neighborhood size (Fig. 10). In the case of $k>1$, a voting of majority decision is made to determine the class of the unknown sample. However, it can be seen from [5], [14], [17] that $k=1$ consistently yields the highest accuracy and was verified by experimentation through the development process. Training the KNN classifier means saving the feature vectors from the training samples as opposed to other classifiers such as Support Vector Machines (SVM) and Artificial Neural Networks (ANN) in which parameters adapt or learn from the training samples.

In comparison to SVM and ANN as classifiers for character recognition, the performance of $\mathrm{KNN}$ is relatively debatable due to the fact that the performance of each classifier is directly related to the chosen training data set. Reference [14] concluded that KNN performed slightly better compared to ANN with multilayer perceptron while [18] showed that ANN is better than KNN at a higher computational cost. Reference [17] observed that SVM and KNN performed slightly better than ANN. Reference [15] showed that SVM performs better than ANN and KNN while the performance of KNN is least among the three. The performance of SVM and ANN becomes more consistent with more training samples, KNNs performance on the other hand, becomes inconsistent early at some point because of statistical redundancy especially to overfitted model with lower values of $k$ and not to mention the computation time will get worse, [14], [18]. Unlike the SVM and ANN, the KNN classifier has more tolerance to nonlinearities caused by a smaller training set. This is very desirable due to the fact that acquiring real-world samples to train the classifier

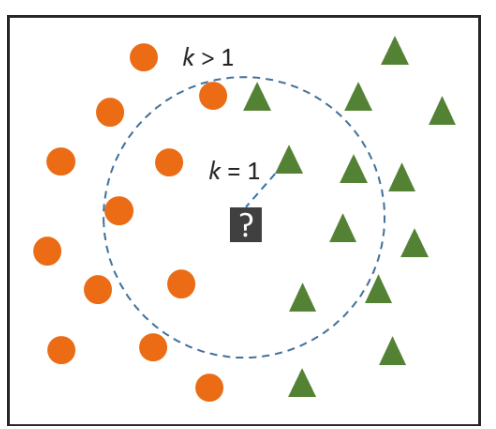

Fig. 10 Illustration of K-nearest neighbor at $k=1$ and $k>1$. 
Table 1 Template space used in KNN.

\begin{tabular}{cccccccc}
\hline Template & $\begin{array}{c}\text { No. of } \\
\text { Fonts }\end{array}$ & $\begin{array}{c}\text { Orient. } \\
1\end{array}$ & $\begin{array}{c}\text { Orient. } \\
2\end{array}$ & $\begin{array}{c}\text { Orient. } \\
3\end{array}$ & $\begin{array}{c}\text { Orient. } \\
4\end{array}$ & $\begin{array}{c}\text { Orient. } \\
5\end{array}$ & Total \\
\hline A-Z & 4 & \multirow{2}{*}{ Normal } & $+5^{\circ}$ & $-5^{\circ}$ & $+15^{\circ}$ & $-15^{\circ}$ & 520 \\
$0-9$ & 5 & & Rot. & Rot. & Skew & Skew & 250 \\
\hline
\end{tabular}

for different conditions is often expensive and sometimes virtually not possible. SVM is a more popular method in character recognition for license plates due to its fast computation speed unlike ANN or deep learning method which is slower and has high computational cost, [16], but SVM also requires big training sample size for consistency over multiclass problems, as with the case of [3] and [9]. The KNN classifier can also obtain a very fast execution speed using an optimal size feature vector and training set that consists the necessary variations. Considering the difficulty of obtaining the training set, KNN will be the best choice.

\subsection{Classification and Confidence Scoring with KNN}

In this method, the nearest neighbor rule will determine the class of the unknown while the neighborhood $k$ of closest matches will be used for confidence scoring of the decision. In the proposed method for confidence scoring, the locality $k$ of nearest neighbors to the unknown sample is chosen by $k<0.5 \min \left(O\left(t_{i}\right)\right)$ such that the function $O(x)$ determines the occurrences of the same class label of templates in the template space and $t_{i}$ is the specific template in the template space.

The template space is generated digitally using a photo editor software due to the lack of complete data samples over the possible orientation of characters. Also, due to the lack of complete data samples over the range of characters, the primitive samples are acquired from several font generator websites and from several fonts in MS Word that resembles very close similarities to that of fonts used in Philippine license plates. The template space is as follows:

From the template space, the neighborhood $k$ for confidence scoring is determined to be 7 where $0.5 \min \left(O\left(t_{i}\right)\right)=$ 8 . Then, each character region is normalized to $16 \times 24$ pixels before subjected to KNN classification and confidence scoring. Due to the non-probabilistic nature KNN classification, there is no inherent method of confidence scoring. A method is devised to evaluate the nearest neighbor decision over each character region and is described as follows:

Let: $N \in \mathbb{R}$, vector of $k$ nearest neighbors

$W=[14,22,17,14,12,11,10]$ where indices $i=1$ to 7

$M \in\{0,1\}^{7}$, vector space containing 0 s and 1 s where first element $M_{1}$ is always 1 .

$$
\begin{aligned}
& M_{i}=\left\{\begin{array}{l}
0, \text { if } N_{i} \neq N_{1} \\
1, \quad \text { else }
\end{array} ; i>1\right. \\
& C_{j}=\sum_{i=1}^{7} W_{i} M_{i} ; j=\text { character index }
\end{aligned}
$$

If there are no elements that matches the nearest neigh-
Table 2 Character domain conversion table.

\begin{tabular}{cccccccccccccc}
\hline INPUT & 0 & 1 & 2 & 3 & 4 & 5 & 6 & 7 & 8 & 9 & & & \\
OUTPUT & $\mathrm{O}$ & $\mathrm{I}$ & $\mathrm{Z}$ & $\mathrm{B}$ & $\mathrm{A}$ & $\mathrm{J}$ & $\mathrm{G}$ & $\mathrm{Y}$ & $\mathrm{B}$ & $\mathrm{B}$ & & & \\
\hline & & & & & & & & & & & & & \\
\hline INPUT & $\mathrm{A}$ & $\mathrm{B}$ & $\mathrm{C}$ & $\mathrm{D}$ & $\mathrm{E}$ & $\mathrm{F}$ & $\mathrm{G}$ & $\mathrm{H}$ & $\mathrm{I}$ & $\mathrm{J}$ & $\mathrm{K}$ & $\mathrm{L}$ & $\mathrm{M}$ \\
OUTPUT & 4 & 8 & 0 & 0 & 6 & 1 & 6 & 8 & 1 & 3 & 8 & 1 & - \\
\hline & & & & & & & & & & & & & \\
\hline INPUT & $\mathrm{N}$ & $\mathrm{O}$ & $\mathrm{P}$ & $\mathrm{Q}$ & $\mathrm{R}$ & $\mathrm{S}$ & $\mathrm{T}$ & $\mathrm{U}$ & $\mathrm{V}$ & $\mathrm{W}$ & $\mathrm{X}$ & $\mathrm{Y}$ & $\mathrm{Z}$ \\
OUTPUT & - & 0 & - & 0 & 8 & 5 & 7 & 0 & 9 & - & 8 & 9 & 2 \\
\hline
\end{tabular}

bor, a score of $14(100 / 7)$ is given as a confidence score to that decision. The rest of weights are derived as follows: $9+F_{i}$ such that $F_{i}$ pertains to the elements of the Fibonacci sequence with index $i>1$. The lowest possible confidence score per decision is 14 and the highest is 100 .

\subsection{License Plate Character String De-Noising}

This stage aims to remove unwanted character regions that segmentation de-noising could not handle. These regions might be side plate borders that are emphasized during binarization which appears at the beginning or the end of a character string, and the center monument image in the old platestyle on complex background (Fig. 7-a) which appears in the middle of the character string. The method of de-noising involves comparison of the string profile with the plate-style and removing the characters in those regions. The unwanted character's confidence score will also be removed so that it will not affect the overall score.

\subsection{License Plate Character Domain Correction}

Character domain is defined in this paper as the subset of characters from the license plate character set that pertains to either the Letter set $\boldsymbol{L}=\{$ "A", "B", "C", ..., "Z" $\}$ or the Numeric set $N=\{“ 0 "$, "1", “2”, . . " $9 "\}$. Character domain correction aims to correct similar characters which are mistakenly classified that should belong to the other domain. Character domain categorization depends on the plate-style. Characters that are misclassified and fall into the wrong character domain are converted if its confidence score is less than 100 using a domain conversion table as follows:

\subsection{Similar Character Discrimination}

Similar characters that are in the same character domain are further identified in this stage. The method of character regional recognition is applied to characters with confidence score of less than 100 to further improve recognition. The same technique is adopted from [10] to feature areas by calculating pixel accumulation in the feature region and setting a threshold so that similar characters could be distinguish. Statistics of similar characters in each domain are as follows:

\subsection{Final Confidence Scoring}

Let: $C$ be the vector of confidence scores of valid character 
Table 3 Statistics of similar characters in the numeric domain.

\begin{tabular}{lllllll}
\hline $\begin{array}{l}\text { Primary } \\
\text { Characters }\end{array}$ & 0 & 3 & 5 & 6 & 7 & 8 \\
\hline Similar & 6 & 8 & 8 & 8 & 2 & 6 \\
Characters & & 9 & 6 & & & 9 \\
\hline
\end{tabular}

Table 4 Statistics of similar characters in the letter domain.

\begin{tabular}{llllllllll}
\hline $\begin{array}{l}\text { Primary } \\
\text { Characters }\end{array}$ & D & F & H & I & L & M & O & P & Q \\
\hline Similar & U & E & W & J & E & H & D & R & D \\
Characters & & & & & & W & Q & & O \\
\hline
\end{tabular}

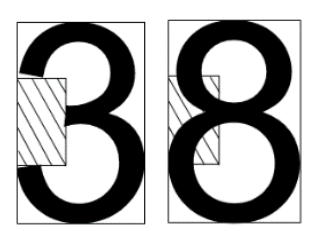

Fig. 11 Feature areas of the characters " 3 " and " 8 " in shaded box.

regions with indices $j=1,2,3, \ldots, \mathrm{N}$ (see Eq. (12))

$$
\begin{aligned}
& C_{\text {ave }}=\frac{1}{\mathrm{~N}} \sum_{j=1}^{\mathrm{N}} C_{j} \\
& C_{\text {final }}=\left\{\begin{array}{lr}
C_{\text {ave }}, & \text { if } \text { char string pattern } \equiv \text { plate style } \\
0.5 C_{\text {ave }}, & \text { else }
\end{array}\right.
\end{aligned}
$$

The final confidence score can strongly indicate the level of certainty if the captured region of interest is a license plate or a false region. Since the plate confidence test will have poor performance on distinctive intermittent patterns that resembles the license plate's horizontal projection, a threshold can be defined to filter these types of false regions.

\section{Testing and Experimentation}

This section aims to evaluate the performance of the proposed ALPR system and individual modules by testing its executional performance and accuracy on the dataset which contains complex scenes. The algorithm is developed in $\mathrm{C}++$ using OpenCV 3.3.0 library and executed in a PC with AMD Ryzen 71700 processor and 16GB DDR4 RAM.

\subsection{Test Dataset}

Scene complexity in this paper is defined by these parameters: Parameter $1(\mathrm{P} 1)$ - number of objects displaying intermittent patterns (e.g., trees, fence, people, and etc.) on background, Parameter 2 (P2) - number of perceivable texture variations (e.g., smooth, contrasting, rough, and etc.), and Parameter 3 (P3) - number of clear texts and characters existing on the image scene other than the license plate. While the first two parameters cannot be exactly determined but a good estimate can be achieved by visual inspection from a human observer.

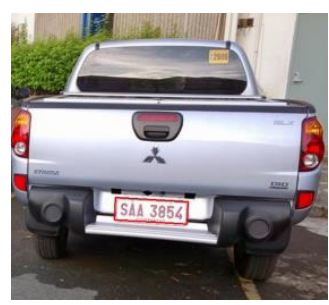

(a)

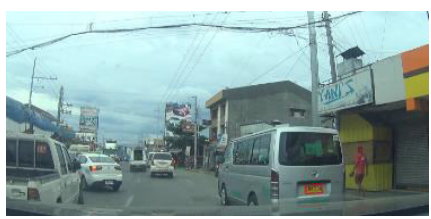

(c)

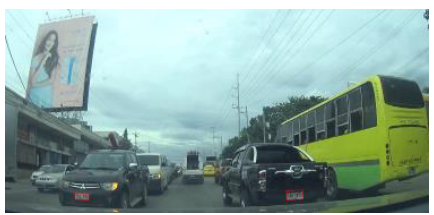

(e)

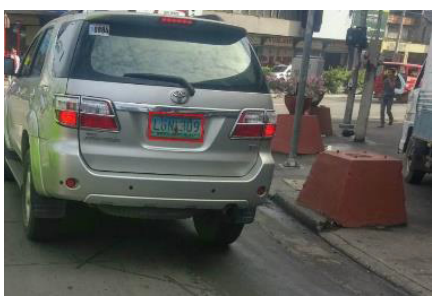

(b)

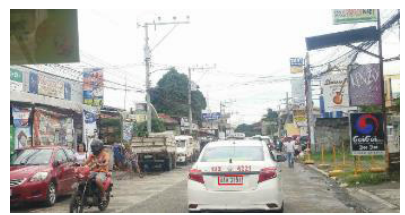

(d)

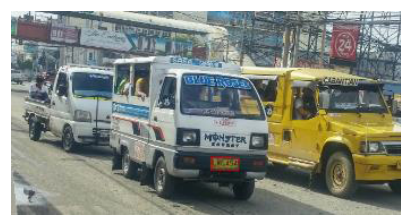

(f)
Fig. 12 (a, b) Images from the non-complex scene. (c, e) Images from the mid-complex scene. (d, f) Images from the high-complex scenes.

Levels of scene complexity are divided into three levels as shown below. The classification for complexity is based on the three parameters A test image to be classified as one of the levels of complexity must satisfy at least two (2) out of three (3) of these conditions as follows:

1. Non-complex: [P1] -1 to 3, [P2] -1 to 3, [P3] -0 to 2

2. Mid-complex: [P1] -4 to $6,[\mathrm{P} 2]-4$ to $6,[\mathrm{P} 3]-3$ to 4

3. High-Complex: $[\mathrm{P} 1]-\geq 7,[\mathrm{P} 2]-\geq 7,[\mathrm{P} 3]-\geq 5$

The test data is composed of 200 images subdivided into: Non-complex scene - 75 test images, Mid-complex scene -80 test images, and High-complex -45 test images. The test data shows that non-complex scenes and midcomplex scenes are far more common than high-complex scenes on practical scenarios during test data gathering.

\subsection{Executional Performance}

The system's executional performance is evaluated using mid-complex test images of varying resolutions. Midcomplex scenes are used because it is more common than non-complex and high-complex in general scenes. Figure 13 shows the execution speed for various resolutions assuming all corners of the image might contain the region of interests for general comparability. In practical situations, the region of interest is bounded only to a part of the image and the whole image size does not need to undergo the process. The system can perform at $30 \mathrm{fps}$ on average using a $640 \times 480(0.3 \mathrm{MP})$ resolution image at $7 \%$ CPU utilization which is practical enough to a number of applications but drops logarithmically as the resolution size increases. The 


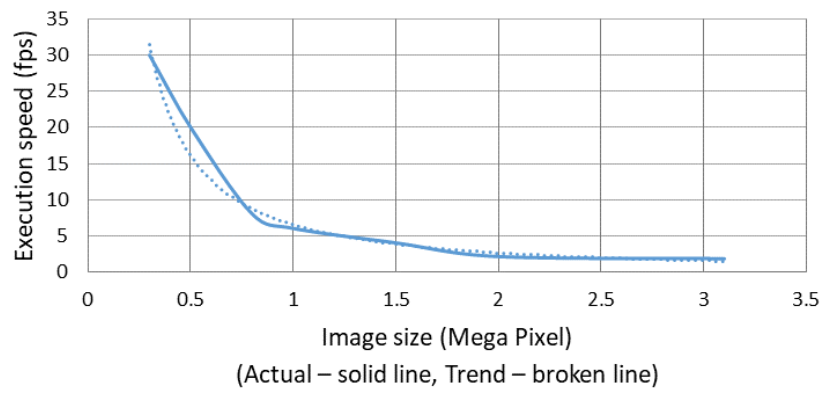

Fig. 13 Execution speed in frames per second (fps) for the proposed ALPR system on mid-complex scenes with respect to the image size.

Table 5 Performance on PC wth AMD Ryzen 71700 Processor and 16GB DDR4 RAM.

\begin{tabular}{cc}
\hline Image size (Mega Pixel) & Ave. CPU Utilization \\
\hline 0.3 & $7 \%$ \\
0.5 & $8 \%$ \\
1.0 & $11 \%$ \\
1.5 & $15 \%$ \\
2.0 & $16 \%$ \\
3.1 & $18 \%$ \\
\hline
\end{tabular}

Table 6 Algorithm performance per level of complexity.

\begin{tabular}{cccccc}
\hline $\begin{array}{c}\text { Scene } \\
\text { Classification }\end{array}$ & $\begin{array}{c}\text { Detection } \\
\text { Rate }\end{array}$ & $\begin{array}{c}\text { Segmentation } \\
\text { Rate }\end{array}$ & $\begin{array}{c}\text { Plate-style } \\
\text { Class. }\end{array}$ & $\begin{array}{c}\text { Recognitio } \\
\text { n Rate }\end{array}$ & $\begin{array}{c}\text { False } \\
\text { Alarm }\end{array}$ \\
\hline Non-complex & $97.33 \%$ & $94.52 \%$ & $97.26 \%$ & $94.03 \%$ & $0.00 \%$ \\
Mid-complex & $90.00 \%$ & $98.61 \%$ & $95.83 \%$ & $92.65 \%$ & $1.39 \%$ \\
High-complex & $100.0 \%$ & $93.33 \%$ & $97.78 \%$ & $92.68 \%$ & $4.44 \%$ \\
\hline
\end{tabular}

Table 7 Overall algorithm performance on the test dataset

\begin{tabular}{cccccc}
\hline $\begin{array}{c}\text { Detection } \\
\text { Rate }\end{array}$ & $\begin{array}{c}\text { Segmentation } \\
\text { Rate }\end{array}$ & $\begin{array}{c}\text { Plate-style } \\
\text { Class. }\end{array}$ & $\begin{array}{c}\text { Recognition } \\
\text { Rate }\end{array}$ & $\begin{array}{c}\text { False } \\
\text { Alarm }\end{array}$ & $\begin{array}{c}\text { Overall } \\
\text { Perf. }\end{array}$ \\
\hline $94.00 \%$ & $95.74 \%$ & $98.40 \%$ & $92.65 \%$ & $1.50 \%$ & $82.05 \%$ \\
\hline
\end{tabular}

Table 8 Performance comparison with existing ALPR on the test dataset

\begin{tabular}{|c|c|c|c|c|c|c|}
\hline $\begin{array}{l}\text { ALPR } \\
\text { System }\end{array}$ & $\begin{array}{l}\text { Philippine } \\
\text { Plate-style }\end{array}$ & $\begin{array}{l}\text { Det. } \\
\text { Rate }\end{array}$ & $\begin{array}{l}\text { Seg. } \\
\text { Rate }\end{array}$ & $\begin{array}{l}\text { Plate- } \\
\text { style }\end{array}$ & $\begin{array}{l}\text { Recog. } \\
\text { Rate }\end{array}$ & $\begin{array}{c}\text { Overall } \\
\text { Perf. }\end{array}$ \\
\hline $\begin{array}{l}\text { Ref. } \\
{[12]}\end{array}$ & YES & $60.00 \%$ & - & - & $81.66 \%$ & $49.00 \%$ \\
\hline $\begin{array}{l}\text { Ref. } \\
\text { [13] }\end{array}$ & NO & $93.00 \%$ & - & - & $76.47 \%$ & $71.12 \%$ \\
\hline $\begin{array}{l}\text { Prop. } \\
\text { ALPR }\end{array}$ & YES & $94.00 \%$ & $95.74 \%$ & $98.40 \%$ & $92.65 \%$ & $82.05 \%$ \\
\hline
\end{tabular}

plate localization stage contributes to over $98 \%$ of the execution time while the character segmentation and recognition only takes less than 2 milliseconds on average per plate. For fast execution on higher resolution, the input image can be sliced into overlapped segments and process in a parallel fashion.

\subsection{Algorithm Performance}

The proposed system is tested in comparison with existing ALPR systems that has already been deployed for commercial use. The test dataset is tested with Ref. [12] which supports several countries including Philippines using their cloud-based demo. Reference [13] is also tested with the test dataset using their cloud-based demo. However, Ref. [13] does not support license plates from Philippines. To be at least on par with the proposed system, Ref. [13] is set to North American setting where a variety of plate-styles would fit the profile of Philippine license plates. During testing, Ref. [12] displayed vulnerability over the increasing level of scene complexity while Ref. [13] and the proposed system is not greatly affected. However, Ref. [13] displayed vulnerability to Philippine plate-style variations even with the plate-style variety offered by the North American setting. The proposed system's processing time is also compared with Ref. [12] and Ref. [13] using the test dataset and found to be much faster by only a small difference.

\section{Conclusion and Discussion}

This paper proposes an approach for detection and recognition of license plates in various scenarios and complex scenes which may pose difficulty to most ALPR designs. Adding to that, the current Philippine licenses plate system poses a challenge of its own because of the multiple platestyles. Using the proposed system on the prepared dataset, accuracy for each module was achieved. A plate detection accuracy of $94.00 \%$, character detection and segmentation accuracy of $95.74 \%$, plate-style classification accuracy of $98.40 \%$, and plate recognition accuracy of $92.65 \%$ with an overall accuracy of $82.05 \%$. Performance of existing ALPR systems are also tested and yielded an overall accuracy of below $72 \%$ on the dataset consisting mostly of complex scenes. A very low false alarm rate was also measured to be $1.50 \%$ on the test dataset which signifies that the proposed method for confidence scoring is effective.

Methods described in this paper are not limited to ALPR and ITS applications. Those methods can be extended on different areas of research. For instance, multisensory detection can be extended to facial recognition problems where frontal, sideward, and facing upward or downward facial features can be detected and classified separately to prevent ambiguity in the classifier. The method of KNN confidence scoring can be extended to topics and researches involving the use of KNN algorithm as a classifier.

\section{Acknowledgments}

This work was supported by Kwangwoon University and by the MISP Korea, under the National Program for Excellence in SW (2017-0-00096) supervised by IITP.

\section{References}

[1] Y. Wen, Y. Lu, J. Yan, Z. Zhou, K.M. von Deneen, and P. Shi, "An Algorithm for License Plate Recognition Applied to Intelligent Transportation System," IEEE Trans. Intell. Transp. Syst., vol.12, no.3, pp.830-845, Sept. 2011.

[2] J.P.D. Dalida, A.-J.N. Galiza, A.G.O. Godoy, M.Q. Nakaegawa, J.L.M. Vallester, and A.R. dela Cruz, "Development of Intelligent Transportation System for Philippine License Plate Recognition," IEEE Region 10 Conference (TENCON), pp.3762-3766, 2016. 
[3] R. Panahi and I. Gholampour, "Accurate Detection and Recognition of Dirty Vehicle Plate Numbers for High-Speed Applications," IEEE Trans. Intell. Transp. Syst., vol.18, no.4, pp.767-779, April 2017.

[4] J. Liu, T. Jin, K. Pan, Y. Yang, Y. Wu, and X. Wang, "An Improved KNN Text Classification Algorithm based on Simhash," IEEE 16th Internation Conference on Cognitive Informatics and Cognitive Computing, pp.92-95, 2017.

[5] T.K. Hazra, D.P. Singh, and N. Daga, "Optical Character Recognition using KNN on Custom Image Dataset," 8th Annual Industrial Automation and Electromechanical Engineering Conference (IEMECON), pp.110-114, 2017.

[6] P. Hurtik and M. Vajgl, "Automatic License Plate Recognition in Difficult Conditions - Technical Report," Joint 17th World Congress of International Fuzzy Systems Association and 9th International Conference on Soft Computing and Intelligent Systems (IFSASCIS), pp.1-6, 2017.

[7] H.-L. Bai, J.-M. Zhu, and C.-P. Liu, "A Fast License Plate Extraction Method on Complex Background," Proc. IEEE International Conference on Intelligent Transportation Systems, vol.2, pp.985-987, 2003.

[8] L.G. Shapiro and G.C. Stockman, Computer Vision, Prentice-Hall, Upper Saddle River, New Jersey, 2001.

[9] O. Bulan, V. Kozitsky, P. Ramesh, and M. Shreve, "Segmentationand Annotation-Free License Plate Recognition with Deep Localization and Failure Identification," IEEE Trans. Intell. Transp. Syst., vol.18, no.9, pp.2351-2363, 2017.

[10] Y.-T. Wang, T.-T. Qin, R.-X. Tian, and G. Yang, "Recognition of License Plate Character Based on Wavelet Transform and Generalized Regression Neural Network," 24th Chinese Control and Decision Conference (CCDC), pp.1881-1885, 2012.

[11] J.-W. Hsieh, S. Yu, and Y.-S. Chen, "Morphology-Based License Plate Detection from Complex Scenes," Proc. IEEE 16th International Conference on Pattern Recognition, vol.3, pp.176-179, 2002.

[12] ANPRonline.Net ${ }^{\mathrm{TM}}$, 2018. Automatic Number Plate Recognition (ANPR) Demo. Retrieved 2018, from https://www.anpronline.net/ demo.html

[13] OpenALPR Technology, Inc. 2018, OpenALPR Cloud API, Retrieved 2018, from http://www.openalpr.com/cloud-api.html

[14] A.R. Quiros, R.A. Bedruz, A.C. Uy, A. Abad, A. Bandala, E. Dadios, and A. Fernando, "A kNN-based Approach for the Machine Vision of Character Recognition of License Plate Numbers," TENCON - IEEE Region 10 Conference, pp.1081-1089, 2017.

[15] K.B. Baiju and K. Sabeerath, "Online Recognition of Malayalam Handwritten Scripts - A Comparison Using KNN, MLP and SVM," International Conference on Advances in Computing, Communication and Informatics (ICACCI), pp.2078-2083, 2016.

[16] C.W. Tan, U.U. Sheikh, and A.A.-H. Ab Rahman, "Improved Optical Character Recognition with Deep Neural Network," IEEE 14th International Colloquium on Signal Processing \& its Applications (CSPA), pp.245-249, 2018.

[17] N.B.A. Hamid and N.N.B.A. Sjarif, "Handwritten Recognition Using SVM, KNN and Neural Network," arXiv:1702.00723v1 [cs.CV].

[18] T. Makkar, Y. Kumar, A.K. Dubey, A. Rocha, and A. Goyal, "Analogizing Time Complexity of KNN and CNN in Recognizing Handwritten Digits," Fourth International Conference on Image Information Processing (ICIIP), pp.1-6, 2017.

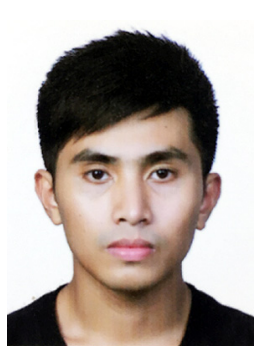

Vince Jebryl Montero received his B.S. degree in Electronics Engineering from Ateneo de Davao University in 2014 and is currently studying for master's degree in Electronics and Comm. Engineering at Kwangwoon University.

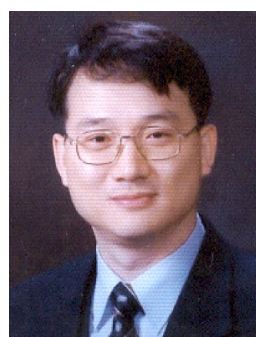

Yong-Jin Jeong received his B.S. degree in Control and Instrumentation Engineering from Seoul National University in 1983. He received his M.S. and $\mathrm{PhD}$ degrees in Electrical and Computer Engineering from University of Massachusetts. He is currently a professor in the Dept. of Electronics and Communications Engineering, Kwangwoon University. 